\title{
Causality in Propagation of a Pulse in a Nonlinear Dispersive Medium
}

\author{
G. S. Agarwal ${ }^{1,2}$ and Tarak Nath Dey $^{1}$ \\ 1 Physical Research Laboratory, Navrangpura, Ahmedabad, India \\ 2 Department of Physics, Oklahoma State University, Stillwater, OK-74078
}

(Dated: August 1, 2018)

\begin{abstract}
We investigate the causal propagation of the pulse through dispersive media by very precise numerical solution of the coupled Maxwell-Bloch equations without any approximations about the strength of the input field. We study full nonlinear behavior of the pulse propagation through solid state media like ruby and alexandrite. We have demonstrated that the information carried by the discontinuity, i.e, front of the pulse, moves inside the media with velocity $c$ even though the peak of the pulse can travel either with sub-luminal or with super-luminal velocity. We extend the argument of Levi-Civita to prove that the discontinuity would travel with velocity $c$ even in a nonlinear medium.
\end{abstract}

PACS numbers: 42.65.-k, 42.50.Gy

The propagation of a pulse of electromagnetic radiation through a linear medium depends critically on the dispersive properties of the medium. Sommerfeld and Brillouin investigated this problem in great detail 1]. They discussed how the group velocity could be very different depending on whether one is working in the region of anomalous dispersion or normal dispersion. Some of these results were verified [2]. Sommerfeld and Brillouin also answered an important question - how information travels through the medium. Interest in this subject has been revived [3, 4, 5, [6] since by using external laser fields one can produce a desired dispersion 7, 8]. Such techniques have, in fact, led to the realization of ultraslow light. Based on an earlier suggestion of Chiao 9], Wang et al. demonstrated the production of superluminal propagation [10]. The propagation of a pulse in a linear medium can also be understood from the interference of various Fourier components present in the input pulse [11]. Stenner et al. were able to demonstrate the causal behavior in the propagation of pulses by studying the propagation of discontinuities in pulse shapes [12].

An important question is - how the discontinuities travel in a nonlinear medium? Needless to say that pulses in a nonlinear medium have been studied quite extensively since the early work of Mc-Call and Hahn 13, 14, 15, 16. In this letter, we examine propagation of discontinuities in a nonlinear medium. We choose solid state materials like ruby and alexandrite. Bigelow et al. studied pulse propagation in such materials and showed the possibility of both sub-luminal and superluminal propagation 17, 18]. These authors specifically concentrated on the nonlinear regime. We use our earlier theoretical models [19] to study the propagation of the front of a pulse and its discontinuities in materials like ruby and alexandrite. We show how the argument of Levi-Civita [Ref.1,p.38] can be extended to prove that the front would travel with velocity $c$ even in a nonlinear medium. This is true despite the fact that the group

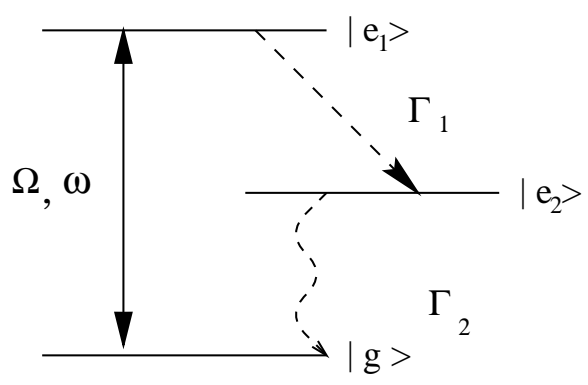

FIG. 1: Schematic diagram of three level system for the ruby crystal.

velocity could be either sub-luminal or super-luminal.

Propagation of pulses with discontinuties in ruby - In order to develop an understanding of causality in pulse propagation through ruby, we consider the model as shown in Fig (11). The figure shows the energy level of chromium ion's $\left(\mathrm{Cr}^{+3}\right)$ in corundum $\left(\mathrm{Al}_{2} \mathrm{O}_{3}\right)$ wherein we denote ground state level ${ }^{4} \mathrm{~A}_{2}$ by $|g\rangle$, the ${ }^{4} F_{2}$ absorption band by $\left|e_{1}\right\rangle$, and the doubly degenerate levels $2 \bar{A}, \bar{E}$ by $\left|e_{2}\right\rangle$. An intense field $\vec{E}(z, t)=\overrightarrow{\mathcal{E}}(z, t) \exp (i k z-i \omega t)+$ c.c. couples states $|g\rangle$ and $\left|e_{1}\right\rangle$ with Rabi frequency $\Omega(z, t)=$ $2 \vec{d}_{1 g} \cdot \overrightarrow{\mathcal{E}}(z, t) / \hbar$, where $\vec{d}_{1 g}$ is the dipole matrix element and $\overrightarrow{\mathcal{E}}$ is the slowly varying envelope of the intense electric field. Here we assume that the carrier frequency of intense field, $\omega$, is in resonance with the frequency of the $|g\rangle \longleftrightarrow\left|e_{1}\right\rangle$ transition. The density matrix equations for the system, as shown in Fig. (11), are given by [19]

$$
\begin{aligned}
& \dot{\rho}_{g g}=2 \Gamma_{2} \rho_{22}+i \frac{\Omega}{2}\left(\rho_{1 g}-\rho_{g 1}\right) \\
& \dot{\rho}_{22}=2 \Gamma_{1} \rho_{11}-2 \Gamma_{2} \rho_{22} \\
& \dot{\rho}_{1 g}=-\Gamma_{1} \rho_{1 g}+i \frac{\Omega}{2}\left(\rho_{g g}-\rho_{11}\right) \\
& \rho_{g g}+\rho_{11}+\rho_{22}=1,
\end{aligned}
$$

where $\rho_{i j}=\left\langle e_{i}|\rho| e_{j}\right\rangle ; i, j=1,2$. Ruby has very strong 
relaxation effects where the dephasing rate $\Gamma_{1}$ is very large compared to population relaxation rate $\Gamma_{2}\left(\Gamma_{1} \gg\right.$ $\left.\Gamma_{2}\right)$. Typically $\Gamma_{1} \sim .15 \mathrm{GHz}$ and $\Gamma_{2} \sim 35 \mathrm{~Hz}$. Under the approximations $\Gamma_{1} \gg \Gamma_{2}, \Omega$, we obtain $\dot{\rho}_{1 g} \sim 0$ and then the evolution equation for the ground state population can be written in the form

$$
\dot{\rho}_{g g}=\left(1-\rho_{g g}\right)-\frac{\Omega^{2}}{4 \Gamma_{1} \Gamma_{2}} \rho_{g g} .
$$

In writing (2) we have used the moving coordinates i.e., the dot denotes the derivative with respect to $2(t-z / c) \Gamma_{2}$. Note that under $\Gamma_{1} \gg \Gamma_{2}, \Omega$ we can set $\rho_{11} \approx 0$. In the slowly varying envelope approximation, we obtain the evolution of the Rabi frequency of an intense field as given by

$$
\frac{\partial \tilde{\Omega}}{\partial z}=-\frac{\alpha_{0}}{2} \tilde{\Omega} \rho_{g g}, \quad \tilde{\Omega}=\Omega / \Omega_{\text {sat }},
$$

where the coupling constant $\alpha_{0}=4 \mathcal{N} \pi \omega\left|d_{1 g}\right|^{2} / c \hbar \Gamma_{1}$ and $\Omega_{\text {sat }}=\sqrt{4 \Gamma_{1} \Gamma_{2}}$. In order to study the causality in the propagation of a pulse through ruby, we consider the shape of the pulse as incident on the input face of the medium given by

$$
\begin{aligned}
\tilde{\Omega}_{i n} & =\tilde{\Omega}^{0} e^{-[\tau / \sigma]^{2}} \quad \tau \leq T \\
& =0 \quad \tau>T .
\end{aligned}
$$

A sharply vanishing amplitude gives rise to the discontinuity in the pulse. Here $\Omega^{0}$ is a real constant indicating the peak amplitude of the pulse and $\sigma$ is the temporal width of the input pulse. Once the shape of the input pulse is specified, the working equations (2) and (3) can be integrated numerically to investigate the evolution of the pulse. We consider all atoms to be initially in the state $|g\rangle$ at $\tau=0$. The length of the medium $\mathrm{L}$ and coupling constant $\alpha_{0}$ are chosen as $9 \mathrm{~cm}$ and $1.17 \mathrm{~cm}^{-1}$ respectively. Fig. (2) depicts the propagation of the input pulse through ruby crystal which is known to exhibit normal dispersion. To prove that the discontinuity of the pulse moves inside ruby with a velocity $c$, numerical integration with high accuracy needs to be performed and the step size of the numerical integration to be taken of the order of $10^{-10}$ in the time domain. This is due to fact that the discontinuity of the pulse takes only $0.3 \mathrm{~ns}$ to traverse a path length of $9 \mathrm{~cm}$ in the medium. We found that the difference in time between the discontinuity of the pulse at output and input ends of the medium is $65 \times 10^{-9}$ in units of $1 / 2 \Gamma_{2}$ by seeing the numerical output as given by table (II), which is not well resolved in Fig. (2). Therefore, the discontinuity of pulse moves inside the ruby crystal with velocity $c$, although, it is evident from Fig. (2) that the peak of the pulse propagates with a subluminal velocity. This conforms with causality in the propagation of information through a nonlinear dispersive medium. We emphasize that in numerical

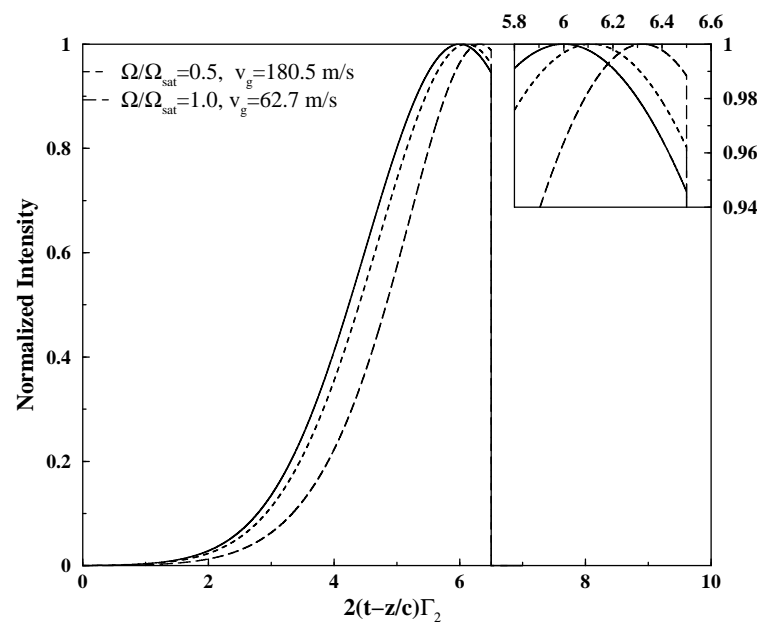

FIG. 2: The solid curve depicts light pulse propagation through a free space with a velocity c. The long-dashed and dashed curves show the light pulse propagating through the medium at different intensities. The discontinuity of the pulse moves with a velocity c irrespective of medium or free space and obeys causality. The temporal width of the pulse is 20 $\mathrm{ms}$ and $1 / 2 \Gamma_{2}=4.45 \mathrm{~ms}$.

TABLE I: Numerical solution of the coupled Maxwell-Bloch equations for the input pulse with peak amplitude $\widetilde{\Omega}^{0}=1$ and discontinuity occurring at 6.5 in units of $1 / 2 \Gamma_{2}$ are given in this table. The path length traversed by the pulse through vacuum as well as the medium is $9 \mathrm{~cm}$. The difference in time for the discontinuity of the pulse to traverse the medium is $65 \times 10^{-9}$ in units of $1 / 2 \Gamma_{2}$. All intensities are normalized to the peak values.

\begin{tabular}{ccc}
\hline \hline $\begin{array}{c}\text { Retarded time } \\
2(t-z / c) \Gamma_{2}\end{array}$ & $\begin{array}{c}\text { Output through } \\
\text { vacuum }\end{array}$ & $\begin{array}{c}\text { Output through } \\
\text { medium }\end{array}$ \\
\hline 6.4999999350 & 0.945844116 & 0.988440243 \\
6.4999999675 & 0.945843694 & 0.988440217 \\
6.5000000000 & 0.945843273 & 0.988440191 \\
6.5000000325 & 0.945842852 & 0.988440165 \\
6.5000000650 & 0.945842430 & 0.988440139 \\
6.5000000975 & 0.000000000 & 0.000000000 \\
\hline \hline
\end{tabular}

simulations we have not used any kind of linearization on the nonlinear equations (2) and (3). Note further that we work at intensities which are comparable to saturation intensities. The information travels in all cases with the velocity $c$ even though the peak position depends on the power of the pulse.

Propagation of pulses with fronts in alexandrite - To prove the causality of pulse propagation in a nonlinear anomalous dispersive medium, we consider alexandrite crystal where reverse saturation absorption is dominant. Reverse saturation absorption produces a narrow antihole in the susceptibility of the probe in the presence of a pump field which can lead to super-luminal prop- 


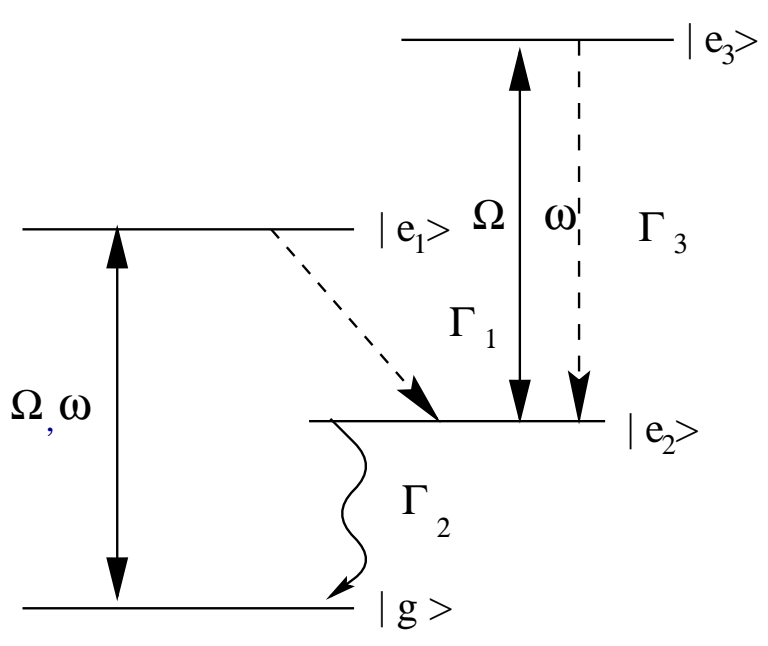

FIG. 3: Four level model for alexandrite crystal.

agation [18]. To investigate the propagation of the discontinuity of the pulse through alexandrite, we refer to the ground state ${ }^{4} \mathrm{~A}_{2}$ by $|g\rangle$, the absorption bands ${ }^{4} \mathrm{~T}_{2}$ and ${ }^{4} \mathrm{~T}_{1}$ by $\left|e_{1}\right\rangle$ and the level ${ }^{2} \mathrm{E}$ by $\left|e_{2}\right\rangle$ as shown in Fig (3). The intense pulse is defined by the electric field, $\vec{E}(z, t)=\overrightarrow{\mathcal{E}}(z, t) e^{-i(\omega t-k z)}+$ c.c., which couples $\left|e_{1}\right\rangle \longleftrightarrow|g\rangle$, and also drives $\left|e_{3}\right\rangle \longleftrightarrow\left|e_{2}\right\rangle$. The density matrix equations for the model as shown in the Fig. (3) are given by [19]

$$
\begin{aligned}
& \dot{\rho}_{g g}=2 \Gamma_{2} \rho_{22}+i \Omega\left(\rho_{1 g}-\rho_{g 1}\right) / 2 \\
& \dot{\rho}_{22}=2 \Gamma_{1} \rho_{11}-2 \Gamma_{2} \rho_{22}+2 \Gamma_{3} \rho_{33}+i \Omega\left(\rho_{32}-\rho_{23}\right) / 2 \\
& \dot{\rho}_{33}=-2 \Gamma_{3} \rho_{33}+i \Omega\left(\rho_{23}-\rho_{32}\right) / 2 \\
& \dot{\rho}_{32}=-\Gamma_{3} \rho_{32}+i \Omega\left(\rho_{22}-\rho_{33}\right) / 2 \\
& \dot{\rho}_{1 g}=-\Gamma_{1} \rho_{1 g}+i \Omega\left(\rho_{g g}-\rho_{11}\right) / 2 \\
& \rho_{g g}+\rho_{11}+\rho_{22}+\rho_{33}=1 .
\end{aligned}
$$

Here we consider that the intense pulse is in resonance with $\left|e_{1}\right\rangle \longleftrightarrow|g\rangle$ and $\left|e_{3}\right\rangle \longleftrightarrow\left|e_{2}\right\rangle$, respectively. We can show that $\dot{\rho}_{32}$ and $\dot{\rho}_{1 g}$ vanish when the approximations $\Gamma_{1}, \Gamma_{3} \gg \Gamma_{2}, \Omega$; are taken into account. Under the same approximations, we obtain the equation for the evolution of ground state population as

$$
\dot{\rho}_{g g}=\left(1-\rho_{g g}\right)-\left|\tilde{\Omega}^{2}\right| \rho_{g g} .
$$

Note that $\rho_{11} \& \rho_{33} \sim 0$ can be easily proved under $\Gamma_{1}, \Gamma_{3} \gg \Gamma_{2}, \Omega$. Therefore, the evolution equation for the slowly varying Rabi frequency of the intense field is given by

$$
\frac{\partial \tilde{\Omega}}{\partial z}=-\frac{\alpha_{0}}{2} \tilde{\Omega} \rho_{g g}-\frac{\tilde{\alpha_{0}}}{2} \tilde{\Omega}\left(1-\rho_{g g}\right)
$$

where $\alpha_{0}$ and $\tilde{\alpha}_{0}$ gives the saturation and reverse saturation, respectively. Shand et al. have shown that, for an excitation wavelength of $457 \mathrm{~nm}$, the excited-state $\left(\left|e_{2}\right\rangle\right)$ absorption cross-section $\left(\sigma_{2} \sim 4.05 \times 10^{-20} \mathrm{~cm}^{2}\right)$

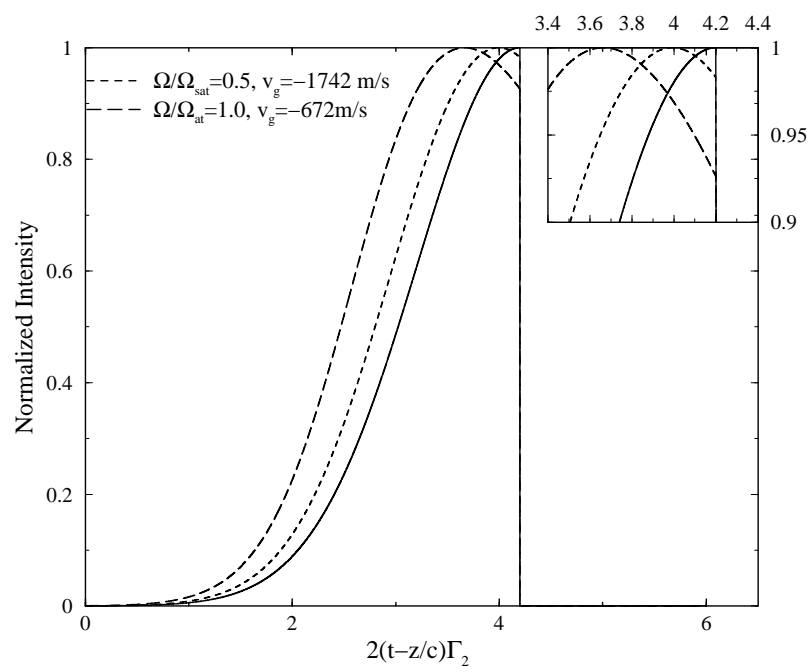

FIG. 4: The solid curve shows light pulse propagating at speed c. The dashed and long-dashed curves depict the propagation of the same pulse through an alexandrite crystal of length $9 \mathrm{~cm}$ with time delays $-51.7 \mu \mathrm{s}$ and $-134 \mu$ s respectively, whereas the discontinuity of the pulse moves with a velocity c. The width $\sigma$ and $1 / 2 \Gamma_{2}$ are $500 \mu$ s and $250 \mu$ s respectively.

exceeds that of the ground state $(|g\rangle)$ absorption crosssection $\left(\sigma_{1} \sim 0.9 \times 10^{-20} \mathrm{~cm}^{2}\right)[20]$. Following these experimental data, we estimate $\left(\tilde{\alpha}_{0} / \alpha_{0}\right) \sim 4$. The numerical integration of coupled Maxwell-Bloch equations (6) and (7) gives the evolution of the input pulse as stated by eq. (4). Here we follow the same numerical integration procedure as stated in the case of ruby. We obtain the time for the discontinuity of the pulse to propagate through alexandrite as well as vacuum to be $12 \times 10^{-7}$ in units of $1 / 2 \Gamma_{2}$ by checking the output of the numerical result (table (III)). This implies that the discontinuity of pulse moves inside the media with a velocity of light in free space $c$. We also found that the group velocity of the input pulse is in the range of superluminal velocity as illustrated in Fig. (4). Therefore, we present a very precise numerical simulation that proves that the discontinuity in a pulse of a electromagnetic radiation cannot propagate faster than the velocity of light $c$ even though medium exhibits nonlinear anomalous dispersion. Consequently, the information carried by the discontinuity cannot propagate with superluminal velocity. It should be borne in mind that the used intensities in the numerical simulation are comparable with the saturation intensities and the solution of the coupled equations (6) and (7) do not use any kind of perturbation. Hence, we have studied the full nonlinear causal behavior of pulses through alexandrite.

Finally we show how the argument of Levi-Civita, as quoted in the book of Brioullion [1], can be extended to prove causality for propagation in a nonlinear medium. Let us consider a plane electromagnetic field say $\hat{x}$ polar- 
TABLE II: This table gives the result of the numerical integrations. The input pulse has peak amplitude $\widetilde{\Omega}^{0}=1$ with the discontinuity at 4.2 in units of $1 / 2 \Gamma_{2}$. It shows that the time taken by the discontinuity of the pulse to propagate through medium as well as vacuum is $12 \times 10^{-7}$ in units of $1 / 2 \Gamma_{2}$.

\begin{tabular}{ccc}
\hline \hline $\begin{array}{c}\text { Retarded time } \\
2(t-z / c) \Gamma_{2}\end{array}$ & $\begin{array}{c}\text { Output through } \\
\text { vacuum }\end{array}$ & $\begin{array}{c}\text { Output through } \\
\text { medium }\end{array}$ \\
\hline 4.2000000 & 0.9999999 & 0.92618654 \\
4.2000003 & 0.9999999 & 0.92618647 \\
4.2000006 & 0.9999999 & 0.92618639 \\
4.2000009 & 0.9999999 & 0.92618632 \\
4.2000012 & 1.0000000 & 0.92618624 \\
4.2000015 & 0.0000000 & 0.00000000 \\
\hline \hline
\end{tabular}

ized and travelling in the $z$-direction so that the electric and magnetic vector have the form $\vec{E}=\hat{x} E(z, t) ; \vec{B}=$ $\hat{y} B(z, t)$. The Maxwell equations then give us

$$
\begin{aligned}
& \frac{\partial E}{\partial z}=-\frac{1}{c} \frac{\partial B}{\partial t} \\
& \frac{\partial B}{\partial z}=-\frac{1}{c} \frac{\partial E}{\partial t}-\frac{4 \pi}{c} \frac{\partial \mathcal{P}}{\partial t}
\end{aligned}
$$

where $\mathcal{P}$ is the nonlinear polarization of the medium. The medium is isotropic and hence the polarization is in $\hat{x}$ direction and is a function of $z, t$. Let $e$ and $h$ be the discontinuities in $\partial E / \partial z$ and $\partial B / \partial z$ at boundary of the wavefront. Note that at the boundary of the wavefront $E$, $B$ and $\partial \mathcal{P} / \partial t$ are continuous. If $v$ represents the velocity with which the discontinuity moves in the direction of its normal then the identity compatibility relations imply that

$$
\text { discontinuity in } \frac{\partial E}{\partial t}=-v e
$$

and

$$
\text { discontinuity in } \frac{\partial B}{\partial t}=-v h \text {, }
$$

This is seen from the equation $E(z+\Delta z, t+$ $\Delta t)-E(z, t)=\Delta t \partial E / \partial t+\Delta z \partial E / \partial z$. Using compatibility relations (910) and Maxwell eqs. (8) we get $v=c$.

In conclusion, we have established by both numerical simulations and analytical methods that the front travels with the velocity of light in free space even in a nonlinear medium.
GSA thanks R. Boyd and M.S. Biegelow for preliminary data on their experiments.

[1] L. Brillouin, Wave propagation and group velocity (Academic, New York, 1960).

[2] S. Chu and S. Wong, Phys. Rev. Lett. 48, 738 (1982).

[3] L. V. Hau, S. E. Harris, Z. Dutton, and C. H. Behroozi, Nature (London) 397, 594 (1999).

[4] D. Budker, D.F. Kimball, S.M. Rochester, and V.V. Yashchuk, Phys. Rev. Lett. 83, 1767 (1999).

[5] M.M. Kash, V.A. Sautenkov, A.S. Zibrov, L. Hollberg, G.R. Welch, M.D. Lukin, Y. Rostovtsev, E.S. Fry, and M.O. Scully, Phys. Rev. Lett. 82, 5229 (1999).

[6] G.S. Agarwal and Tarak Nath Dey, Phys. Rev. A 68, 063816 (2003).

[7] S.P. Tewari and G.S. Agarwal, Phys. Rev. Lett. 56, 1811 (1986); D.F. Phillips, A. Fleischhauer, A. Mair, R.L. Walsworth, and M.D. Lukin, Phys. Rev. Lett. 86, 783 (2001); C. Liu, Z. Dutton, C.H. Behroozi, and L.V. Hau, Nature (London) 409, 490 (2001).

[8] S.E. Harris, Phys. Rev. Lett. 62, 1033 (1989); H. Kang, G. Hernandez, and Y. Zhu, Phys. Rev. Lett. 93, 073601 (2004).

[9] A.M. Steinberg and R.Y. Chiao, Phys. Rev. A 49, 2071 (1994); R.Y. Chiao and A.M. Steinberg, in Progress in Optics, Ed. E. Wolf (Elsevier, Amsterdam, 1997), pg. 345.

[10] L.J. Wang, A. Kuzmich, and A. Dogariu, Nature(London) 406, 277 (2000).

[11] A. Dogariu, A. Kuzmich, H. Cao, and L.J. Wang, Opt. Exp. 8, 344 (2001).

[12] M.D. Stenner, D.J. Gautheir, and M.A. Neifeld, Nature(London), 425, 695 (2003).

[13] S. L. McCall and E. L. Hahn, Phys. Rev. Lett. 18, 908 (1967).

[14] R. Grobe, F. T. Hioe, and J. H. Eberly, Phys. Rev. Lett. 73, 3183 (1994).

[15] F. T. Hioe and R. Grobe, Phys. Rev. Lett. 73, 2559 (1994).

[16] G. S. Agarwal and J.H. Eberly, Phys. Rev. A 61, 013404 (2000).

[17] M.S. Bigelow, N.N. Lepeshkin, and R.W. Boyd, Phys. Rev. Lett. 90, 113903 (2003).

[18] M.S. Bigelow, N.N. Lepeshkin, and R.W. Boyd, Science, 301, 200 (2003).

[19] G.S. Agarwal and Tarak Nath Dey, Phys. Rev. Lett. 92, 203901 (2004).

[20] M.L. Shand, J.C. Walling, and R.C. Morris, J. Appl. Phys. 52, 953 (1981). 